\title{
CONCEPTUALIZACIONES METAFÓRICAS Y RECURSOS DE FORMULACIÓN EN NARRACIONES DE PACIENTES CON MIGRAÑA
}

\author{
GUIOMAR ELENA CIAPUSCIO \\ Universidad de Buenos Aires-CONICET
}

\section{RESUMEN}

En este trabajo analizo un conjunto de narraciones sobre episodios críticos que realizan personas que sufren de migraña. La voz de los pacientes ha adquirido en los últimos años mayor relevancia, después de haber sido largamente postergada en el campo de la salud. Las investigaciones lingüísticas sobre el discurso de los pacientes pueden aportar al conocimiento y comprensión de la enfermedad, incluso a su diagnóstico, como lo han mostrado, entre otros, las investigaciones sobre las enfermedades cardíacas y convulsivas realizadas por equipos alemanes e ingleses (Gülich y Schöndienstt, 1999; Schwabe et al., 2008; Schwabe et al., 2007). En este artículo, tomando como perspectiva la teoría conceptual de la metáfora y la teoría de la producción discursiva, presento un análisis de un corpus de narraciones de pacientes, en las que las personas exponen y explican a conocidos y familiares sus ataques de migraña: me interesa avanzar en el conocimiento del modo en que estos pacientes conceptualizan y transmiten su enfermedad y su síntoma principal: el dolor de cabeza. El análisis de las contribuciones de los pacientes se detiene tanto en los aspectos cualitativos del dolor como en la descripción de su intensidad, que es particularmente rica en procedimientos y recursos expresivos y conduce, en ocasiones, a la tematización de sentimientos de miedo y pánico.

PALABRAS CLAVE: metáfora, procedimientos de formulación, expresiones del dolor, recursos de intensificación, narraciones de enfermedad.

\section{AbSTRACT}

This paper discusses a set of narratives on critical episodes by people affected by migraine. The voice of patients has acquired higher relevance in recent years, after being long postponed in the field of medicine. Linguistic research on patients' discourse can contribute to the knowledge on and understanding of this disorder, and even to its diagnosis, as shown among others by the researches on cardiac and convulsive illnesses carried out by German and British teams (Gülich and Schöndienstt, 1999; Schwabe et al., 2008; Schwabe et al., 2007). In this article I 
present, from the perspective of conceptual metaphor theory and of discursive production theory, an analysis of a corpus of patient narratives in which people explain their migraine attacks: I am interested in expanding the knowledge on how these patients conceptualize and transmit their illness and its main symptom, i.e. headache. The analysis of patients' contributions focuses both on the qualitative aspects of pain and on the description of its intensity, which is particularly rich as regards expressive procedures and resources and leads, occasionally, to the thematization of feelings of fear and panic.

KEYWORDS: metaphor, formulation procedures, expressions of pain, intensification resources, illness narratives.

\section{INTRODUCCIÓN}

La migraña es definida desde el punto de vista médico como un trastorno neurológico caracterizado por ataques de cefalea, hipersensibilidad a los estímulos visuales, auditivos, olfatorios y cutáneos, náuseas y vómitos. Existen diferentes tipos de migraña: básicamente, con y sin aura, aunque se distinguen dentro del primer tipo varias subcategorías (cfr. IHS 2013); es de subrayar que la constelación de síntomas difiere de paciente en paciente, así como también la intensidad de los mismos. Este mal, que afecta aproximadamente al $12 \%$ de la población occidental ${ }^{1}$, con mayor incidencia en las mujeres, es de naturaleza crónica, puede afectar a los pacientes de manera muy frecuente (la migraña crónica se define por una frecuencia de ataques de, al menos, 15 días en el mes) ${ }^{2}$ y produce limitaciones severas para llevar una vida normal. Según estudios realizados en Estados Unidos, la prevalencia de la migraña crónica aumenta a lo largo de la adolescencia, tiene picos en la mediana edad y luego disminuye a partir de los 50 años; la prevalencia más elevada se observa en las mujeres de 18 a 49 años. Se trata de una enfermedad que se encuentra sub-diagnosticada aún en países muy desarrollados (Sator, 2011), y frecuentemente es subestimada y minimizada incluso por parte de los médicos.

En este trabajo analizo un conjunto de narraciones sobre episodios críticos que realizan personas que sufren de migraña. Las narraciones sobre la enfermedad son objeto de interés creciente: la sociología de la medicina, la psicología, la antropología y el análisis del discurso reconocieron hace décadas la importancia de los aspectos comunicacionales e interaccionales en el campo de la salud (por ej. Atkinson, 1995; Atkinson et al., 2001, entre muchos otros); más tarde, han surgido corrientes dentro de la medicina

\footnotetext{
${ }^{1}$ Datos de Intramed: <http://www.intramed.net/contenidover.asp?contenidoID=83600 $>$.

${ }^{2}$ Schwedt (2014).
} 
que ponen en primer plano el relato de la enfermedad (Charon, 2006). A lo largo de las últimas décadas, la sostenida demanda social respecto de la necesidad e importancia de considerar y tomar en cuenta las vivencias de los propios pacientes ha conducido al surgimiento de diversas iniciativas dentro del campo de la salud, para mejorar la formación médica en aspectos comunicacionales, pero también para sensibilizarlos y aumentar la conciencia sobre el valor de la palabra del enfermo en el diagnóstico y el tratamiento: cursos y talleres de medicina narrativa, producción de literatura especializada sobre relatos de pacientes ${ }^{3}$, sitios temáticos en Internet, en los cuales los pacientes pueden obtener información fiable sobre su mal y dialogar con otros pacientes sobre sus vivencias, alternativas de tratamiento y curación ${ }^{4}$, importantes proyectos de investigación y reuniones de carácter interdisciplinario sobre las narrativas de enfermedad son cada vez más frecuentes ${ }^{5}$. La voz de los pacientes ha adquirido en los últimos años mayor relevancia, después de haber sido largamente postergada en el campo de la salud. Las investigaciones lingüísticas sobre el discurso de los pacientes pueden aportar al conocimiento y comprensión de la enfermedad, su diagnóstico y tratamiento, como lo han mostrado, por ej., las investigaciones sobre las enfermedades cardíacas y convulsivas realizadas por equipos alemanes e ingleses (Schwabe et al., 2008; Schwabe et al., 2007).

Este trabajo se enmarca en un proyecto de investigación más amplio ${ }^{6}$, uno de cuyos objetivos específicos consiste en estudiar y analizar desde el punto de vista formal y funcional los procedimientos y recursos de formulación que emplean pacientes y médicos para comunicar, en diferentes contextos y géneros, temáticas vinculadas a la enfermedad. Se trata de un proyecto de investigación lingüística, que cuenta con la asesoría externa de especialistas. En este artículo, tomando como perspectiva la teoría conceptual de la metáfora y la teoría de la producción discursiva, presento un

${ }^{3}$ En el ámbito argentino es de destacar la labor de la iniciativa de Intramed (<http://www. intramed.net/>), que convoca regularmente a escritores de renombre para componer relatos ficcionales sobre las vivencias de distintas enfermedades (por ejemplo, La piedra de la cordura: historias sobre enfermedades mentales; Permiso para morir, entre otros, publicados por Ediciones Intramed).

4 Algunos ejemplos son la iniciativa Dipex, en Inglaterra (Personal experiences of health and illness, <http://www.dipex.org>), que se ha extendido a otros países como Japón, Corea y Alemania (ver "Krankheitserfahrungen.de" -Experiencias de salud, enfermedad y medicina-, financiada por el estado alemán).

${ }^{5}$ Por ejemplo, el encuentro internacional e interdisciplinario "Illness narratives in practice", realizado en el Instituto de Psicología de la Universidad de Freiburg en el mes de junio de 2015 (<www.narratives-in-practice.unifreiburg.de>).

6 "La comunicación de la medicina: procedimientos textuales y léxico-gramaticales de la explicación, el diagnóstico y la recomendación” (ANCYT-PICT 2120-2013). 
análisis de un corpus de narraciones de pacientes, en las cuales las personas exponen y explican a conocidos y familiares sus ataques de migraña: me interesa avanzar en el conocimiento del modo en que estos pacientes conceptualizan y transmiten su enfermedad y su síntoma principal: el dolor de cabeza.

La estructura del trabajo es la siguiente: en primer lugar presento de manera sucinta los fundamentos teóricos que dan marco a este análisis y los aspectos metodológicos. A continuación, describo en líneas generales cómo las personas presentan sus ataques de migraña. A continuación, me concentro en las conceptualizaciones metafóricas del proceso de la migraña y sus recursos expresivos, y seguidamente, en su síntoma más característico: la cefalea aguda. El análisis de las contribuciones de los pacientes se detiene tanto en los aspectos cualitativos del dolor como en la descripción de su intensidad, que es particularmente rica en procedimientos y recursos expresivos: el carácter agudo de la cefalea conduce, como se verá, a la tematización de sentimientos de miedo y pánico. Finalmente, recapitulo y valoro los resultados obtenidos.

\section{FundAMENTOS TEÓRICOS Y METODOLÓGICOS}

El marco teórico que sirve de base a estas indagaciones es necesariamente heteróclito, debido al énfasis empírico que las motiva. Por un lado, la teoría conceptual de la metáfora (formulada y ampliada en sucesivos trabajos de Lakoff y Johnson, 1991, 1999; Lakoff y Turner, 1989; Lakoff, 2008, entre otros). En los últimos años, en esta línea se ha investigado intensamente el campo de las metáforas para la expresión de emociones: por ejemplo Kövecses $(2000,2008)$ se ha dedicado a estudiar cómo se conceptualizan en distintas lenguas las emociones como la ira, el amor, la felicidad, el miedo, el dolor; también Semino (2010) y Díaz Vera (2012), entre otros investigadores, han producido aportes sustanciales al conocimiento de las conceptualizaciones y expresiones lingüísticas del dolor y la emoción. A diferencia de estos trabajos, que focalizan la atención en el sistema lingüístico, priorizando el acercamiento introspectivo, lexicográfico o la extracción aislada de elementos lingüísticos de corpus textuales, mi atención se dirige a conversaciones naturales, en las que los interlocutores llevan a cabo actividades lingüísticas para describir y reconstruir narrativamente sus episodios de migraña. Transmitir la experiencia subjetiva de la enfermedad y los dolores que esta causa es una tarea compleja y difícil; los pacientes tropiezan de manera recurrente con dificultades para verbalizar y explicar a los interlocutores sus sensaciones y vivencias, situación que, como lo han mostrado algunas investigaciones previas (Gülich y Furchner, 2002), 
incluso suelen tematizar mediante estructuras lingüísticas relativamente estandarizadas (es difícil de describir, cómo explicarlo) y recursos aproximativos de distinto tipo, como por ejemplo, hedges.

Por esta razón, la segunda vertiente teórica en la que me oriento son los diferentes trabajos sobre las actividades de producción discursiva llevados a cabo por Gülich y Kotschi (1995) que se sustentan en la idea central de que producir discurso es llevar a cabo, de manera progresiva e interactiva, un trabajo que conlleva un esfuerzo cognitivo y temporal (Antos, 1982), que deja huellas en la superficie textual (hecho especialmente evidente en la oralidad). Sobre la base del estudio pormenorizado de esas huellas -en términos más técnicos, marcadores- en amplios corpus de datos orales, Gülich y Kotschi (1995) identificaron y caracterizaron distintos procedimientos de formulación del discurso, que se revelan como regulares y "metódicos", como por ejemplo, las reformulaciones y los comentarios metadiscursivos. También se aprovechan aquí los desarrollos de estos trabajos sobre los procedimientos de ilustración, entre los que se incluye la metáfora conceptual (Brünner y Gülich, 2002; Gülich, 2003), que luego fueron profundizados analítica y empíricamente en diferentes campos de aplicación: para el español, en la comunicación experto-lego (Ciapuscio, 2003; Ciapuscio, 2007); en lengua alemana, los desarrollos realizados en el marco de los proyectos dirigidos por Gülich sobre las enfermedades convulsivas (Gülich y Schöndienst, 1999; Gülich y Furchner, 2002; y especialmente Surmann, 2005 y Gülich, 2007). Existe un conjunto de investigaciones específicas sobre el discurso de pacientes y médicos sobre el dolor, llevadas a cabo en la Universidad de Viena (Menz et al., 2010), y en este mismo marco, la investigación realizada por Sator (2011) sobre las entrevistas en la ambulancia de dolor de cabeza, realizadas por médicos a pacientes a los efectos de diferenciar el tipo de dolor de cabeza y encaminar el diagnóstico y tratamiento ${ }^{7}$.

El corpus, recogido en la ciudad de Buenos Aires, está conformado por seis conversaciones privadas de duración variable: por un lado, pacientes de migraña -de entre 22 y 60 años de edad-, quienes son los que monopolizan la palabra en estos intercambios, y por el otro, personas allegadas (conocidos, amigos, familiares) ${ }^{8}$. Se trata en todos los casos de un contexto de conversación de considerable cercanía interpersonal y relaciones de

${ }^{7}$ Cabe mencionar que existen trabajos relevantes acerca de narrativas sobre migraña desde la perspectiva de la antropología social en el ámbito latinoamericano; especialmente pertinentes son las contribuciones de Del Mónaco (por ej. 2013), en los que pueden encontrarse datos etnográficos de valía.

${ }^{8} \mathrm{Mi}$ agradecimiento a las personas que colaboraron con esta investigación, especialmente a los pacientes, cuyos nombres han sido modificados para mantener el anonimato. 
simetría entre los interlocutores. En todos los casos se solicitó a los pacientes que explicaran y narraran sus ataques de migraña, que describieran sus síntomas y su curso, de manera libre, sin limitaciones temporales. Las conversaciones fueron grabadas con audio y transcriptas según convenciones que figuran al final de este artículo.

El análisis, de orden inductivo y ejemplar, sigue la orientación teórica escogida: las contribuciones de los hablantes se analizan de manera secuencial y se concentra en las formulaciones que van realizando en colaboración interactiva: el foco se coloca en la descripción del proceso de ataque de la migraña y del dolor de cabeza.

\section{ANÁlisis}

\subsection{La migraña como proceso}

Los pacientes describen los episodios de migraña en términos de un proceso progresivo, que se inicia con una serie de síntomas y sentimientos anticipatorios, luego se instala, aumenta, alcanza un climax -el dolor agudo y el sentimiento de invalidación total- y tiene una fase de resolución. Para ello, recurren a diferentes técnicas narrativas (narración episódica, narración iterativa, escenificaciones, etc., cfr. Gülich et al., 2003), a las que solo se aludirá en el comentario de los ejemplos, pero que ameritan un análisis más detallado que sobrepasa los límites de este trabajo.

Las experiencias de la enfermedad difieren entre sí, esencialmente porque el ataque puede o no estar acompañado de auras, trastornos gastrointestinales, de foto y fonofobia ${ }^{9}$. Común a todas las exposiciones es el carácter muy agudo de los dolores de cabeza. En algunos pacientes el proceso se inicia con trastornos visuales -algunos experimentan auras, trastornos de audición (por ej. molestias respecto del exterior como los ruidos)-, irritación ante demandas de comunicación, dolores en distintos lugares de la cabeza -base, costado, frente, ojos-, que se incrementan hasta hacerse en todos los casos "intolerables", "agudos", "tremendos" ${ }^{10}$. Además, otros mencionan náuseas y vómitos, que suelen marcar el fin del ataque. Acostarse, aislarse, encerrarse a oscuras, no moverse, son alternativas a las que recurren para atemperar los síntomas del mal.

9 “ $[\ldots]$ las circunstancias que propician su aparición son variadísimas y, además, hasta donde pude comprobar, no existen dos migrañosos iguales. Los dolores se revelan incomparables” (Gianera, 2015). También Sacks (1995).

${ }^{10}$ Las expresiones que cito entre comillas provienen en su totalidad del corpus bajo análisis. 
Varios de los pacientes destacan el carácter integral del episodio (en el sentido de que afecta todo el cuerpo) y rechazan la idea de la migraña como un mero dolor de cabeza. Por otra parte, destacan el carácter inmotivado e imprevisto, como lo muestran los ejemplos (1) y (2):

(1) Mariano (70 años)

1 no tiene relación con nada de lo que uno haga. o sea que uno no se

2 puede predisponer para que le venga el ataque. uno está tranquilamente.

3 haciendo cualquier cosa de:: y de repente, empieza a tener trastorno de la visión

4 que le anuncia, que le avisa que las próximas cinco horas va a sufrir

(2) Floria (22 años)

1 a veces estoy tranquila mirando tele

2 y pUn' me agarra la migraña

La recurrencia crónica de los ataques de migraña explica el conocimiento detallado y mayormente preciso del proceso que vuelcan los pacientes en sus contribuciones. Además, suelen tematizar en sus descripciones y narraciones sentimientos de angustia y de miedo, que explican a partir de diferentes factores asociados con este mal: la falta de conocimiento de sus causas, el carácter inmotivado o repentino de los ataques, la intensidad del dolor, el no poder detener el curso de los ataques y también la incomprensión social e incluso médica sobre el mal (cfr. Del Mónaco, 2013).

\subsection{Conceptualización metafórica: procedimientos y recursos de ilustración}

Como lo han señalado numerosas investigaciones previas, el carácter subjetivo de la vivencia de la enfermedad y la dificultad de elaborar, describir y transmitir esas experiencias explican que los pacientes recurran de manera frecuente a la metáfora y la analogía. Según la teoría conceptual, la metáfora es un mecanismo esencial del sistema cognitivo, que nos permite comprender un dominio en términos de otro: así conceptualizamos la vida como si se tratara de un viaje, las discusiones como guerras, las ideas como alimentos, las organizaciones sociales como plantas o vegetales, etc. También las emociones y las enfermedades y sus síntomas encuentran su vehículo en el recurso a la conceptualización metafórica: Surman (2005), en su trabajo sobre la metáfora en el discurso de personas con enfermedades convulsivas, concluye que las "metáforas proporcionan una salida al inconveniente de la indescriptibilidad" (mi traducción), es decir, de la dificultad o imposibilidad de describir con palabras los síntomas y sentimientos que frecuentemente experimentan los pacientes (ibídem: 116). 


\subsubsection{Las fases de la migraña}

Los pacientes de migraña focalizan en las distintas fases de sus ataques y las conceptualizan mediante distintas metáforas, que verbalizan con un esmerado trabajo de formulación, en el que emplean un repertorio rico y variado de recursos, como se verá en los ejemplos más abajo.

\section{La fase anticipatoria}

La fase anticipatoria de la migraña que, según las estadísticas, experimentan un $80 \%$ de los pacientes y que se revela en variados síntomas premonitorios, aparece tematizada en este pequeño corpus mediante metáforas de orden meteorológico, como una amenaza de lluvia o de tormenta. Véase el tramo inicial de una descripción:

(3) Ninoska (55 años)

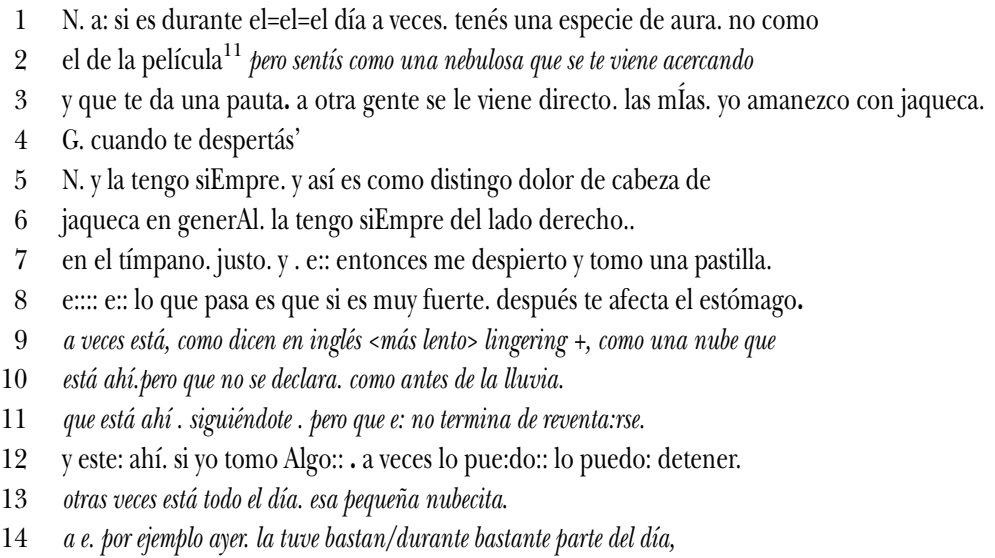

La llegada de la migraña se formula mediante una comparación: como una nebulosa (línea 2) que se acerca paulatinamente y "que avisa" al paciente de la proximidad del ataque. Ninoska precisa su ubicación (la tengo siempre en el lado derecho, en el tímpano, justo). A continuación, elabora y amplía la metáfora de la nube para explicar los diferentes cursos que puede tomar la migraña: primero, introduce la palabra del inglés lingering $^{12}$, cuyo

${ }^{11} \mathrm{~N}$ se refiere a una conocida película argentina titulada "El aura" (2005) (<https://www. youtube.com/watch?v=UIRagZcdFXk>). tionary).

To linger: demorar, quedarse, tardar en partir (Simon and Schuster International Dic- 
significado explica sirviéndose nuevamente de la analogía con el mismo dominio fuente (como una nube que está ahí pero que no se declara); esta metáfora es reformulada inmediatamente, mediante una expansión, en la que se incrementa la ontologización en el sentido de una personificación: como antes de la lluvia, que está ahí, siguiéndote pero no termina de reventarse. La reiteración de la construcción está ahi (líneas 9 y 10) expresa el estado latente y amenazante de la migraña -todavía no disparada- que la paciente formula en términos de una posible lluvia (que no termina de reventar) que puede detenerse con ayuda de medicación. La experiencia reiterada de la posibilidad del ataque expresada mediante la metáfora de la nube sirve también para ilustrar en las líneas 13 y 14 otras circunstancias en las que la migraña persiste como amenaza (otras veces está todo el día esa pequeña nubecita), hecho que se ejemplifica con un caso concreto (por ejemplo ayer, la tuve bastan/durante bastante parte del día). La paciente-como la mayoría de los hablantes del corpus- emplea en este fragmento el esquema de la narración iterativa, caracterizada por el tiempo verbal en presente, la recurrencia de adverbios y sintagmas temporales como siempre (líneas 5, 6), a veces, otras veces (12 y 13), para cerrar con un episodio particular (ayer la tuve bastan/durante bastante parte del día, línea 14).

Otra paciente presenta el estado previo a la llegada de la migraña con la analogía de una tormenta incipiente:

(4) Juliana (28 años)

1 cuando empezás a sentir los síntomas. que como es esto

2 de que a veces es un malestar general. uno no se da cuenta tan fácil.

3 cuando está por venir. e:l=la la migraña.

4 si la/ más o menos la encontrás . o sea si en el momento estoy consciente.

5 de que estoy teniendo dolor de cabe:za. me tomo algo y listo.

6 pero como arranca con un malestar

7 de que no sé si me bajó la presión. si tengo un poco de náuseas

8 . entonces si es algo del estó:mago.

9 si es porque. no termino de entender bien. me duele un poco la cabeza.

10 a veces . no no me doy cuenta de que todo eso. va a llegar después a la migraña.

11 pero sí. es como toda una cosa prEvia que se empieza a preparar.

12 es como va a venir la tormenta y y empiezan pequeñas cosas.

13 y uno si es sensible se da cuenta. no sé. cómo corre el viento,

14 cómo se empiezan a poner las nubes., es lo mismo.

15 son muchas cosas. un/ en conjunto.

16 que llevan a que después es la migraña.

En los tramos iniciales del ejemplo (4) Juliana manifiesta dificultades para reconocer el inicio del ataque de migraña, caracterizado como un malestar general. En las líneas 11 y 12 introduce la analogía con el estado 
previo al inicio de una tormenta, en el que ocurren varios eventos anticipatorios (la dirección del viento, el estado de las nubes); en la línea 14 reafirma la validez absoluta de su analogía (es lo mismo).

\section{El desarrollo de la migraña}

El carácter paulatino y progresivo del proceso de la migraña se ilustra muy bien en el siguiente fragmento, que procede de la entrevista con Floria, quien alterna los tiempos presente e imperfecto, que expresan el carácter recurrente del ataque:

(5) Floria

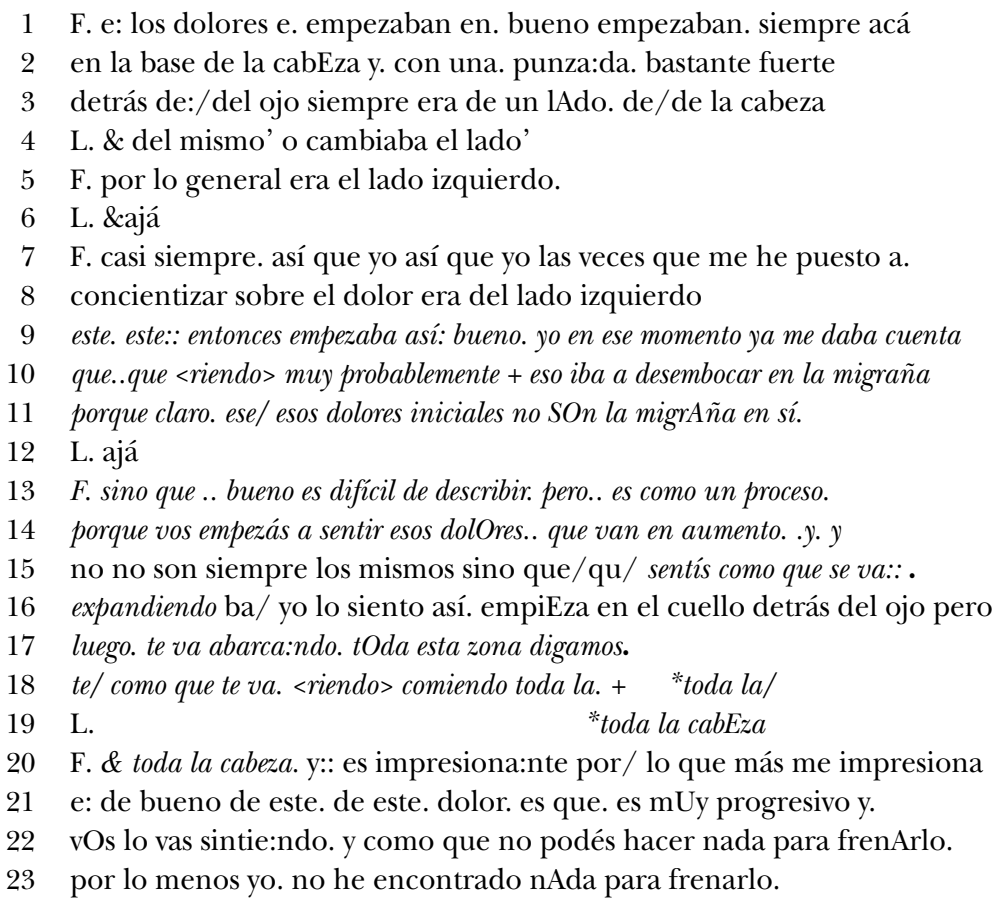

Como todas las personas entrevistadas, Floria comienza por localizar el lugar en el que empieza la migraña y presenta el dolor como una punzada bastante fuerte (línea 2), es decir, como un dolor agudo y repentino, similar a un pinchazo. Luego, precisa que esos dolores iniciales constituyen los anuncios de la migraña próxima a desencadenarse: (líneas 9 y 10: yo en ese momento y a me daba cuenta que... que <riendo> muy probablemente + eso iba a desembocar en la migraña). Mediante el pronombre neutro eso (en línea 10), 
retoma todo el segmento anterior -el comienzo y la localización del dolorpara, a continuación, mediante una construcción adversativa, aclarar que esos dolores iniciales no son la migraña en sí (porque claro. ese/ esos dolores iniciales no SOn la migrAña en sí.). En la línea 13 intenta continuar con la construcción adversativa ( sino que...), que interrumpe con una estructura preformada, frecuente en corpus sobre enfermedades convulsivas (Gülich y Schöndienst, 1999), que expresa la dificultad de verbalización: bueno es difícil de describir. A continuación, el trabajo de formulación se concentra en la explicación del carácter progresivo de los dolores: fundamentalmente se expresa mediante una cadena de reformulaciones: ver líneas 14-17: empezás a sentir esos dolOres. que van en aumento/ sentís como que se va:: . expandiendo/ luego. te va abarca:ndo. tOda esta zona digamos. A continuación, conceptualiza la expansión y el progreso del dolor mediante una comparación (una animización), que señala mediante la risa: es como que te va comiendo toda la cabeza (el interlocutor interviene activamente en la construcción de esta analogía, como se ve en la línea 19): detrás de la expresión metafórica puede reconocerse la metáfora "el dolor es una entidad externa agresiva”, que se reitera en las narraciones de los pacientes, como veremos más adelante.

\subsubsection{La conceptualización y expresión del dolor agudo de cabeza}

El punto más intenso del proceso de migraña es el dolor de cabeza muy agudo, lo cual testimonian las contribuciones de todos los pacientes. Los sujetos conceptualizan y expresan el pico de dolor de la migraña mediante metáforas y comparaciones, por un lado, y por el otro, mediante un repertorio variado de estructuras y recursos de formulación, orientados a verbalizar sus experiencias del dolor. Me centraré en esta sección en dos aspectos sobresalientes de las presentaciones de los pacientes: la calidad y la intensidad del dolor.

En cuanto a la calidad del dolor, se pueden identificar conceptualizaciones metafóricas recurrentes en las expresiones de los pacientes (en todos los casos la fuente del dolor es un instrumento o un agente externo). Los ejemplos (6) y (7) ilustran la conceptualización del dolor en términos de instrumentos externos: en el primer caso, filoso, cortante; en el segundo, opresivo:

(6) Mariano

1 la migraña es un dolor mUy agudo. es un dolor.. este:. treme:ndo un dolor que no te

2 deja: . que no te da tregua... es como si uno tuviese de golpe un cuchIllo.

3 clavado en la cabeza, 
En este momento de su exposición, el hablante está tratando de explicar la diferencia entre un dolor de cabeza corriente y el dolor que causa la migraña; su diferenciación se basa en la intensidad extrema que tiene este último. Para ello realiza una descripción que va aumentando en el grado de dramatismo, primero, a base de un adjetivo de emoción y luego dos construcciones de sentido negativo (tremendo, no te deja: no te da tregua). La descripción alcanza su climax con la introducción de la comparación: como si uno tuviese de golpe un cuchIllo. clavado en la cabeza.

(7) Claudia (50 años)

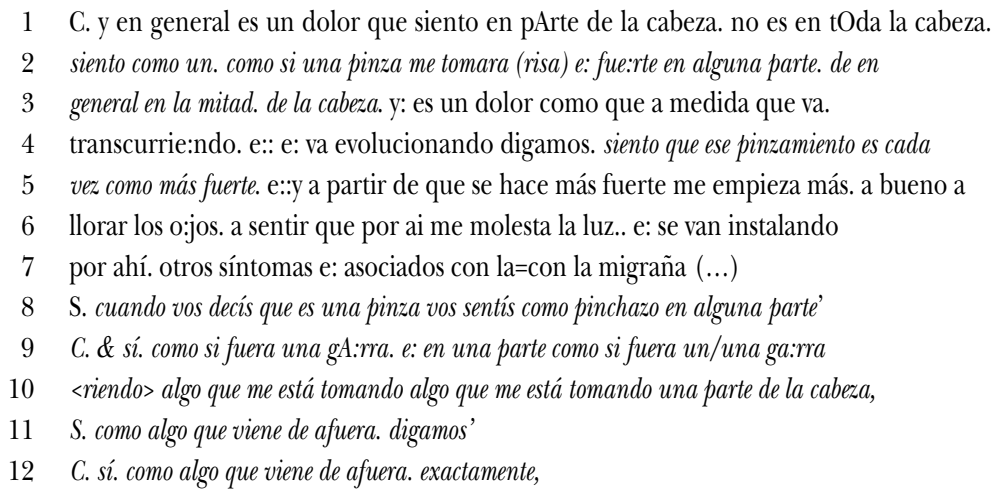

El ejemplo (7) muestra un esforzado trabajo de formulación respecto de la metáfora "el dolor es un instrumento externo" (considérese la reformulación en línea 2: siento como un. como si una pinza...). En un primer momento, la paciente presenta la metáfora en términos de una pinza que va apretando en forma progresiva y cada vez más aguda una parte de su cabeza. Ante la pregunta de su interlocutora (línea 8), vos sentís como pinchazo en alguna parte', reelabora la metáfora apelando a la analogía con una garra (y de este modo el instrumento se convierte en un miembro de un animal) y reafirma la percepción del carácter externo del origen del dolor (línea 12, sí. como algo que viene de afuera. exactamente).

En el cuadro 1 pueden visualizarse las conceptualizaciones metafóricas y en la columna de la derecha expresiones extraídas del corpus que las realizan.

Estos resultados coinciden con los hallazgos de Kövecses para el dolor en general (2008), por lo que -coincidiendo con este autor- reafirman la hipótesis de la validez interlingüística; también se corresponden con los resultados obtenidos por Surman (2005: 167), para el caso de pacientes con epilepsias focales. Como puede verse en la columna de la derecha, las expresiones metafóricas contienen nombres que significan el carácter 
CUADro 1. Conceptualizaciones y expresiones metafóricas para el dolor agudo de cabeza

\begin{tabular}{|c|c|}
\hline Dolor de cabeza & Formulaciones \\
\hline "El dolor es un instrumento filoso" & $\begin{array}{l}\text { la migraña es un dolor mUy agudo. es un dolor. } \\
\text { este: treme:ndo ... es como si uno tuviese de } \\
\text { golpe un cuchIllo. clavado en la cabeza }\left(\mathrm{M}^{13}\right)\end{array}$ \\
\hline \multirow{2}{*}{$\begin{array}{l}\text { "El dolor es un instrumento } \\
\text { opresivo" }\end{array}$} & $\begin{array}{l}\text { Siento como si una pinza me tomara; ese } \\
\text { pinzamiento es cada vez más fuerte }(\mathrm{C})\end{array}$ \\
\hline & $\begin{array}{l}\text { habría que agarrar una morsa y apretar asi } \\
\text { (gesto), con una morsa (A) }\end{array}$ \\
\hline \multirow{3}{*}{$\begin{array}{l}\text { "El dolor es un entidad } \\
\text { (animal/humana) hostil, } \\
\text { amenazante" }\end{array}$} & $\begin{array}{l}\text { como si fuera una garra en una parte.. como si } \\
\text { fuera un/una garra, algo que me está tomando } \\
\text { una parte de la cabeza }(\mathrm{C})\end{array}$ \\
\hline & Algo que me va comiendo toda la cabeza $(\mathrm{F})$ \\
\hline & $\begin{array}{l}\text { Un dolor punzante que no te deja que no le } \\
\text { prestes atención, que está ahí y te dice: mirá que } \\
\text { estoy acá }(\mathrm{F})\end{array}$ \\
\hline
\end{tabular}

agudo del dolor (un cuchillo, una pinza, una morsa) y construcciones verbales hiperbólicas (te va comiendo la cabeza), o que transmiten hostilidad o configuran en el contexto actos amenazantes (mirá que estoy acá).

Además de las expresiones metafóricas, la cualidad del dolor es descripta por los hablantes mediante una amplia gama de recursos de formulación, esencialmente adjetivos y verbos, mediante los que comunican aspectos específicos de la experiencia dolorosa. En coincidencia con los resultados obtenidos por Deppermann (2003: 173), además de transmitir las cualidades de dolor de manera general, los pacientes de este corpus tienen a tematizar los efectos emocionales y las consecuencias en la capacidad de funcionar en la vida práctica. En el cuadro 2 he consignado una muestra de expresiones lingüísticas relevadas para describir el dolor, ordenadas según cualidades sensoriales, emocionales o afectivas, de grado y de "efectos en el sujeto": cientes.

${ }^{13}$ Las letras en mayúsculas corresponden a las letras iniciales de los nombres de los pa- 
CUADRO 2. Recursos de formulación para describir el dolor agudo de cabeza

\begin{tabular}{|c|l|l|l|l|}
\hline $\begin{array}{l}\text { Clases de } \\
\text { palabras }\end{array}$ & $\begin{array}{l}\text { Cualidades } \\
\text { sensoriales }\end{array}$ & $\begin{array}{l}\text { Cualidades } \\
\text { emocionales/ } \\
\text { afectivas }\end{array}$ & Grado & $\begin{array}{l}\text { Efectos } \\
\text { en el sujeto }\end{array}$ \\
\hline Adjetivos & $\begin{array}{l}\text { Agudo, } \\
\text { punzante } \\
\text { continuo, } \\
\text { progresivo }\end{array}$ & $\begin{array}{l}\text { Desesperante, } \\
\text { impresionante, } \\
\text { agobiante, } \\
\text { insoportable, } \\
\text { feo, raro }\end{array}$ & $\begin{array}{l}\text { Tremendo, } \\
\text { terrible, } \\
\text { intenso }\end{array}$ & $\begin{array}{l}\text { Invalidante, } \\
\text { reinvalidante, } \\
\text { incapacitante }\end{array}$ \\
\hline \multirow{6}{*}{ Verbos } & $\begin{array}{l}\text { Me late } \\
\text { la cabeza }\end{array}$ & $\begin{array}{c}\text { No te da } \\
\text { tregua }\end{array}$ & $\begin{array}{c}\text { Me explota } \\
\text { la cabeza } \\
\text { Me estrujan } \\
\text { la cabeza }\end{array}$ & $\begin{array}{c}\text { no podés hacer } \\
\text { noda } \\
\text { no podés trabajar } \\
\text { no ni abrir } \\
\text { los ojos } \\
\text { no podés frenarlo }\end{array}$ \\
& & & & \\
\hline
\end{tabular}

Las conceptualizaciones y expresiones de los pacientes coinciden en general con las de las taxonomías del dolor que se han establecido en distintas instituciones y organizaciones médicas, para diagnosticar y diferenciar las dolencias asociadas con el dolor de cabeza; en efecto, las categorías del cuadro 2, exceptuando las que refieren al impacto de la enfermedad en la vida diaria de los pacientes, coinciden en buena parte con las de la conocida taxonomía de la McGill University, traducida a varias lenguas y, en términos generales, con diversos cuestionarios y esquemas de diagnóstico que se utilizan clínicas y hospitales de diferentes lugares del mundo ${ }^{14}$.

La descripción de la intensidad del dolor es particularmente relevante en las contribuciones de los pacientes, no solo por su omnipresencia en las conversaciones sino porque, además, la descripción del grado extremo del dolor coincide con la expresión de sentimientos de temor y angustia. Estos sentimientos son especialmente tematizados por las pacientes más jóvenes (Floria y Juliana, 22 y 28 años respectivamente): en las narraciones y descripciones de estas pacientes, los motivos de este sentimiento son variados: miedo a los medicamentos, miedo al empeoramiento de la enfer-

${ }^{14}$ Ver por ejemplo el cuestionario para pacientes de la clínica Comprehensive Headache Center Anesthesia, Critical Care and Pain Medicine Beth Israel Deaconess Medical Center, ubicado en Boston, EE.UU. <http://www.bidmc.org/ /media/Files/CentersandDepartments/ AnesthesiaCriticalCareandPainManagement/Headache\%20Center/PatQuestionnaire.pdf $>$. 
medad con el paso del tiempo, miedo a la ocurrencia del ataque y miedo a la intensidad misma del dolor. Especialmente prominente es el último, véase el siguiente ejemplo:

(8) Floria

1 F. hm.. para mí. en el caso del=del tratamiento social es como. como te digo.

2 no' <impostando la voz> sos exagerada. dejá de decir boludeces.+ siEmpre.

3 siEmpre es la misma respuesta. y nO te crEen. es como que no te crEen.

4 este. e. incluso le explicás . a:. este: a mi hermAna.

5 que mi hermana nos ve a mi mamá y a mí.

6 y: y te das cuEnta que dicen <impostando la voz> a. sí sí. + pero. no. como que vos

7 le tenés que poner un nOmbre. ponele tengo::. angInas. ay ahí sí.

8 <impostando la voz>ay ay anginas +. y quizás eso es . una. pavA:da. en rea/

9 porque yo he tenido anginas. REgrosas. y la verdAd que me REba:nco una

10 angina.me banco una otItis. me banco una conjuntivitis. porque he tenido todas

11 esas.me las REba:nco entonces son como nA:DA en relación con el dolO=r.

12 que te ocasiona la migraña.. es un dolor ade:ntro que ... nAda.

13 no se pUEde.. yo siempre digo. cuando me dolía la ore:ja y hacía asi: y

14 al menos sentís que. no sé Algo. que/ se te pAsa un poqui:to. te duE:le.

15 el Ojo. qué se yo. pero en la/ade:ntro de la cabeza

16 es mu:y desesperA:nte. a mí a veces. m: incluso me DA mIEdo porque...es muy/

17 no sé.no sabés si te: si te puede pasar Algo. si no te puede pasar nA:da

18 L. claro=claro

19 F. porque es como te digo eso. t/ además te ocasiona un montón de

20 cOsas que no son normales

En este fragmento Floria recurre de manera reiterada al recurso de la escenificación (Gülich et al., 2003): mediante esta técnica de la narración los eventos se presentan como si estuvieran sucediendo en ese mismo momento, por lo cual es frecuente el empleo del discurso directo, mediante el cual se ponen en escena las distintas voces de los participantes que intervienen en el suceso narrado (aquí muy evidente porque $\mathrm{F}$ además imposta las voces de otros -la madre, la hermana-), lo cual otorga un carácter vívido y dramático a la narración.

En las líneas 2-11, la señora F establece una comparación entre la migraña, que caracteriza como una enfermedad frecuentemente minimizada (ver línea 3: no te creen, es como que no te creen) y otras enfermedades (anginas, otitis, conjuntivitis), que gozan de reconocimiento y comprensión social. Para describir la intensidad del dolor de la migraña, la hablante en la línea 9 argumenta que ella ha sufrido episodios agudos de angina (he tenido anginas REgrosas) y que sin embargo esos dolores son incomparables con la migraña: para ello utiliza un esquema de formulación que he llamado estructuras hiperbólicas, y que aparecen muy frecuentemente en esta función descriptiva: son como nada en relación con el dolor que te ocasiona la 
migraña. La intensidad de sus sentimientos y vivencias se expresa, además, con la anteposición del prefijo $r e$ a adjetivos y verbos, un procedimiento muy generalizado en el español rioplatense actual. A continuación, intenta describir el dolor pero interrumpe la formulación es un dolor adentro que... no se puede para nuevamente recurrir a un procedimiento de comparación, que califica como de uso rutinizado en sus descripciones (yo siempre digo, línea 13): el contraste lo realiza entre órganos "exteriores" y la cabeza: tras la enumeración ( te duele la oreja, te duele el ojo...), establece la contraposición con la estructura adversativa pero en la /adentro de la cabeza es muy desesperan$t e$, que describe el impacto emocional del dolor. Es en este punto (líneas 16-17) en el que aparece tematizado el miedo: a mi a veces. m: incluso me DA mIEDO porque... es muy/no sé. no sabés si te: si te puede pasar ALgo. si no te puede pasar nA:da.

El siguiente fragmento ilustra el empleo de diferentes recursos de la intensificación:

(9) Adriano (47 años)

1 el dolor. ya no me acuerdo si era continuo o pulsado, o las dos cosas.

2 e: viste por las arterias, por el tema de la dilatación del por la/ los latidos.

3 pero era muy muy intenso. y desesperante, y más de una vez este:

4 por la cantidad de horas que llevaba el dolor y era:: daban ganas de=de

5 arrancarse el ojo. viste. sí la solución era sacarse el ojo. porque sale del fondo del

6 ojo, la..como si fuera en la parte de atrás del ojo. y el cerebro, la cabeza. todo

Como puede verse en el ejemplo, el carácter agudo del dolor se expresa mediante la combinación de distintos recursos intensificadores (cuantificadores muy, muy, y adjetivos -muy intenso y desesperante (línea 3) - y el climax se alcanza mediante la expresión hiperbólica, daban ganas de=de arrancarse el ojo, la solución era sacarse el ojo. Los hablantes suelen combinar este recurso con estructuras superlativas, un tipo de estructura preformada (Gülich, 2007) de alto grado de convencionalización (casos como llorar de dolor, morir de dolor, desmayar de dolor). Véase el ejemplo 10:

\section{(10) Floria}

1 F. sí. a mí me ha pasado a veces. e e: de llorar del dolor porque es horrible.

2 es a la noche cuando me acosta:ba era horrible.

3 no. no lo puedo creer que me esté pasando esto. me daba bronca

4 L. y si tomabas aspiri:na'

5 F. \& no nada. nada. es como un carameli:to. nada nada e:: terrible

En (10) pueden observarse distintos recursos para expresar la intensificación: la locución superlativa -llorar de dolor-, las repeticiones (es horrible) era horrible), la estructura preformada (no lo puedo creer que me esté pasando 
esto) y, finalmente, ante la pregunta de L. ¿y si tomabas aspirina? la paciente en primer lugar niega con una hipérbole el efecto de los remedios para el dolor de cabeza ( son nada) e introduce una analogía de significado irónico -la aspirina es como un caramelito- que logra la hipérbole para negar todo efecto benefactor y significar así la imposibilidad de detener la intensidad del dolor (lo cual remarca con la reiteración nada: nada y el calificativo terrible).

Véase por último este fragmento de la contribución de Mariano, que emplea en pocas líneas un variado repertorio de recursos de intensificación, haciendo un uso notorio de estructuras hiperbólicas:

(11) Mariano

1 es $m U y$ mUy invalidante es la migraña. tremEnda. es aparte. es un dolor' que:..

2 no $\mathrm{t}$ /= no podés nI pe:nsar. viste que uno por lo general cuando se acuesta a

3 dormir.tiene un momento previo al/a dormir que uno piensa qué se yo. lo que le

4 pasó durante el día o cualquier cosa. . te duele hasta pensAr. es un dolor

5 treme:ndo. y: y esas cuatro o cinco horas que te duele te parece que/ una

6 semana. (.. ?) no= no termina más. cada minuto de ese dolor es es treme:ndo

La intensidad del dolor se expresa nuevamente sobre la base de repeticiones de cuantificadores ( $m u y$, línea 1 ), de adjetivos emocionales (tremen$d a$, tremendo, tremendo líneas 1,5 y 6 ), estructuras negativas con significado superlativo como no podés ni pensar (línea 2), hasta construcciones hiperbólicas: te duele hasta pensar (línea 4). La intensidad del dolor se conceptualiza además a través de la percepción temporal subjetiva, determinada por aquella intensidad: por un lado, el tiempo se extiende de manera desmesurada; el paciente experimenta unas horas como si se tratara de una semana (líneas 5 y 6 ), por el otro, la agudeza del dolor no cesa ni mengua en ningún momento (cada minuto de ese dolor es tremendo, línea 6).

En el siguiente ejemplo Juliana está narrando dos episodios que califica como los más graves que ha padecido: en ambos, la migraña desencadena el pánico:

\footnotetext{
me pasó esas dos veces. me empecé a sentir mAl. no sé si es como que sentí que me bajó la presión que no me podía concentrar en las cosas. que estaban a mi alrededor .y empezaba levemente a doler la cabeza.. después. me empezó a doler la cabeza cada vez mÁs.y. apareció e: me agarran náuseas. el tema es que si no tomo nAda y me dejo estar.el dolor de cabeza. persiste y cada vez es $p E O R$. es pEOr. las náuseas son peo:res. y. mi contacto con el entorno es peor.

no puedo :. no puedo pensar. no puedo escuchar a nAdie.

8 me siento $\mathrm{mAl}$. necesito estar aislAda. hasta el momento en que . si $=$ si sigue

9 empeorA:ndo. las dos veces que me pasó yo estaba lejos de cAsa.. una en el

10 trabajo y otra en la facultad.y me agarra como el miE:do. de. quiero llegar ya a
} 
11 mi casa. me siento mAl.o sé si es una especie de ataque de pánico. pero más o

12 menos es como que digo <riendo> me voy a morir + estoy lejos de casa.

13 necesito estar en mi casa yA. transportarme. no quiero hablar con nA:die.

14 quiero que me pase el dolor de cabe:za.

En esta descripción es notorio el recurso a estructuras preformadas convencionalizadas, que suelen adoptar la forma de la repetición para expresar el incremento del malestar y del dolor: en las líneas 4 y 5 expresa que si no recurre a medicación en el momento adecuado, el dolor persiste y aumenta: a partir de allí emplea una serie de repeticiones de la construcción es peor/son peores (líneas 5 y 6) y el paralelismo con la frase verbal no puedo (línea 7, no puedo pensar, no puedo escuchar a nadie). El punto extremo de la intensidad del dolor es el miedo (línea 10): me agarra como el miedo, que se explica en términos de desesperación por estar en su casa (necesito estar en mi casa $y A$, transportarme). El miedo enseguida se insinúa en términos de pánico (no sé si es un ataque de pánico) y se explicita finalmente como un miedo a morir (línea 12: es como que digo me voy a morir), motivado en el carácter muy agudo del dolor.

Por último, presento un ejemplo en el que la paciente expresa un tipo de miedo diferente, que, a diferencia del anterior, tiene un carácter permanente: es el que la paciente experimenta ante la posibilidad del ataque. Este fragmento corresponde al tramo final de la descripción de Floria:

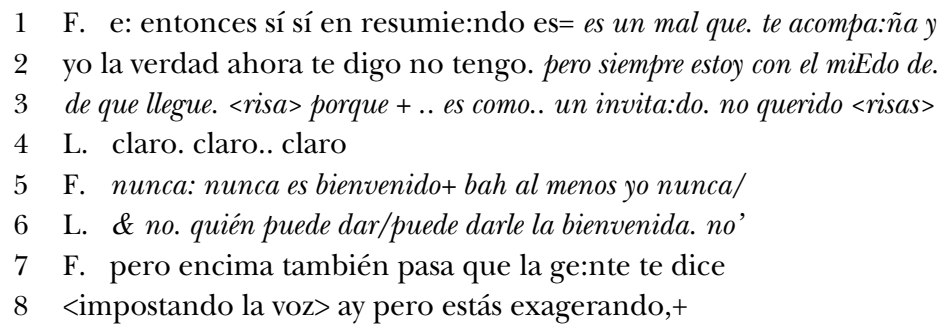

Como se observa en la primera línea, la paciente hace referencia a la migraña como enfermedad crónica, introduciendo una antropomorfización (es un mal que te acompaña) que va incrementando en su intervención: ese mal le produce un miedo permanente -una inquietud instalada- (siempre estoy con el miedo de que llegue), que expresa expandiendo la metáfora mediante una comparación: es como un invitado no querido que nunca es bienvenido, analogía que ratifica la interlocutora con una pregunta retórica (quién puede darle la bienvenida). Este tipo de miedo podría caracterizarse como crónico. 


\section{RECAPITULACIÓN Y PANORAMA}

En este trabajo he presentado un análisis exploratorio de narraciones, centrado en cómo los pacientes conceptualizan, elaboran y comunican sus ataques de migraña y su síntoma principal -la cefalea aguda- en el marco de la teoría conceptual de la metáfora y los estudios sobre los procedimientos de formulación discursiva. Del análisis de los datos, puede concluirse que, para relatar y describir sus ataques, los hablantes recurren a conceptos metafóricos regulares (la migraña como un evento meteorológico -lluvia o tormenta-) y el dolor de cabeza como provocado por un instrumento externo (punzante o presionante) o por un agente animado hostil. Para transmitir el proceso de la migraña y la calidad e intensidad del dolor de cabeza -su síntoma principal- llevan a cabo un rico trabajo de formulación, que incluye metáforas, comparaciones, reformulaciones y distinto tipo de estructuras preformadas. La expresión de la intensidad máxima del dolor se realiza a través de un repertorio variado de recursos, que incluye la repetición de elementos, el empleo de estructuras preformadas, cuantificadores, estructuras hiperbólicas y locuciones superlativas.

En síntesis, mediante la ilustración con casos ejemplares he intentado brindar un cuadro inicial de algunos recursos prominentes que emplean los pacientes para describir sus ataques; esa descripción a menudo incluye la tematización de diferentes tipos de miedos: por un lado, un miedo episódico, motivado por la intensidad del dolor, cuyo desenlace incierto produce, en el caso de las pacientes jóvenes, desesperación y pánico; por el otro, un miedo crónico, ocasionado por el carácter recurrente que caracteriza la enfermedad.

El análisis lingüístico y discursivo de las narraciones de los pacientes de migraña sobre su enfermedad -tomando como base corpus de datos más amplio y controlado- podría ser un insumo de interés para mejorar los instrumentos diagnósticos para la diferenciación de los tipos de migraña. Por ejemplo, podría indagarse la posibilidad de que existan ciertas correspondencias entre los tipos de migraña y el empleo de determinados conceptos metafóricos y la preferencia por determinados recursos de formulación (como el empleo o no de expresiones estereotipadas o estandarizadas, cfr. Gülich, 2007). Más allá de la posibilidad más o menos cierta de aportar al conocimiento de esta enfermedad crónica, frecuentemente sub-diagnosticada y subestimada, el análisis del discurso de los pacientes echa luz sobre los procedimientos metafóricos y recursos lingüísticos para la expresión del dolor agudo en español. 


\section{BIBLIOGRAFÍA}

ANTos, G. (1982): Grundlagen einer Theorie des Formulierens: Textherstellung in geschriebener und gesprochener Sprache, Tubinga, Niemeyer.

AtKinson, P. (1995): Medical Talk and Medical Work: the Liturgy of the Clinic, Londres, Sage Publications

Atkinson, P., Coffey, A., Delamont, S., Lofland, J. y Lofland, L. (eds.) (2001): Handbook of Etnography, Thousand Oaks, CA, Sage Publications.

BRÜNNER, G. y GÜLICH, E. (2002): "Verfahren der Veranschaulichung in der Experten-Laien-Kommunikation”, en G. Brünner y E. Gülich (eds.), Krankheit verstehen. Interdisziplinäre Beiträge zur Sprache in Krankheitsdarstellungen, Bielefeld, Aisthesis Verlag, págs. 17-93.

Charon, R. (2006): Narrative Medicine: Honoring the Stories of Illness, Oxford, Oxford University Press.

Ciapuscio, G. (2003): "Formulation and reformulation procedures in verbal interactions between experts and semi-laypersons", Discourse Studies, 5 (2), págs. 207-233.

- (2007): "Acciones de calificación en conferencias de divulgación científica", Revista Internacional de Lingüistica Iberoamericana (RILI), vol. V, nº 1 (9), págs. 59-77.

Del Mónaco, R. (2013): "Dolor crónico y narrativa: experiencias cotidianas y trayectorias de atención en el padecimiento de la migraña”, Physis. Revista de Saúde Coletiva, 23, págs. 489-510.

Deppermann, A. (2003): "Wenn Semantik zum praktischen Problem wird: Divergierende Schmerzkonzepte von Ärztin und Patientin in der psychosomatischen Exploration”, Psychotherapie und Sozialwissenschaft, 3, V \& R, Göttingen, págs. 165-182.

Díaz VERA, J. (2012): "When pain is not a place: Pain and its metaphors in late middle English medical texts", Onomázein, 26 (2), págs. 279-308.

Gianera, P. (2015): "El dolor de cabeza: manual de uso", La Nación [31-01-2015].

GÜLICH, E. (2003): "Conversational techniques used in transferring knowledge between medical experts and non-experts”, Discourse Studies, 5 (2), págs. 235263.

- (2005): "Unbeschreibbarkeit: Rhetorischer Topos - Gattungsmerkmal - Formulierungsressource", Gesprächsforschung-Online-Zeitschrift zur verbalen Interaktion, Ausgabe 6, págs. 222-244 (<www.gespraechsforschung-ozs.de>).

- (2007): "Volle Palette in Flammen'. Zur Orientierung an vorgeformten Strukturen beim Reden über Angst”, Psychotherapie \& Sozialwissenschaft, 1, págs. 59-87.

- y Kotschi, Th. (1995): "Discourse production in oral communication", en U. Quasthoff (ed.), Aspects of oral communication, Berlín, Mouton de Gruyter, págs. 30-66. 
— y SCHÖNDIENST, M. (1999): “'Das ist unheimlich schwer zu beschreiben'. Formulierungsmuster in Krankheitsbeschreibungen anfallskranker Patienten: differential diagnostische und therapeutische Aspekte", Psychotherapie und Sozialwissenschaft, 1, págs. 199-227.

- y Furchner, I. (2002): "Die Beschreibung von Unbeschreibbarem. Eine konversationsanalytische Annäherung an Gespräche mit Anfallskranken”, en I. Keim y W. Schütte (eds.), Soziale Welten und kommunikative Stile, Tubinga, Narr, págs. 161-186.

—, Schöndienst, M. y Surmann, V. (2003): "Schmerzen erzählen Geschichten Geschichten erzählen Schmerzen”, Psychotherapie und Sozialwissenschaft, 3, V \& R, Göttingen, págs. 219-248.

IHS (2013): "International Classification of Headache Disorders (3rd edition beta version)”, Cephalgia, 33 (9), págs. 629-808, Londres, Sage Publications.

Kövecses, Z. (2000): Metaphor and Emotion, Cambridge, Cambridge University Press.

- (2008): "The conceptual structure of happiness and pain", en C. Lascaratou, A. Despotopoulou y E. Ifantidou (eds.), Reconstructing Pain and Joy: Linguistic, Literary and Cultural Perspectives, Cambridge, Cambridge Scholars Publishing, págs. 17-33.

LAKoff, G. (2008): "The neural Theory of Metapher", en R. Gibbs (ed.), The Cambridge Handbook of Metaphor and Thought, Cambridge, Cambridge University Press, págs. 17-38.

- y Turner, M. (1989): More than Cool Reason. A Field Guide to Poetic Metaphor, Chicago, The University of Chicago Press.

— y Johnson, M. (1991): Metáforas de la vida cotidiana, Madrid, Cátedra.

- (1999): Philosophy in the Flesh. The embodied Mind and its Challenge to Western Thought, Basic Books, Perseus Books Group.

Menz, F. J., Lalouschek, M., Sator, K. y Wetschanow (2010): Sprechen über Schmerzen. Linguistische, kulturelle und semiotische Analysen, Duisburg, Universitätsverlag Rhein-Ruhr.

SACKS, O. (1995): Migraine. Revised and Expanded, London, Picador.

SATOR, M. (2011): Schmerzdifferenzierung. Eine gesprächsanalytische Untersuchung ärztlicher Erstgespräche an der Kofpschmerzambulanz, Viena, V \& R unipress.

SEmino, E. (2010): "Descriptions of pain, metaphor and embodied simulation", Metaphor and Symbol, 25 (4), págs. 205-226.

Schwabe, M., Reuber, M., SchÖndienst, M. y Gülich, E. (2008): "Listening to people with seizures: How can linguistic analysis help in the differential diagnosis of seizure disorders?”, Communication and Medicine, 5 (1), págs. 59-72.

Schwabe, M., Howell, S. y Reuber, M. (2007): "Differential diagnosis of seizure disorders: A conversation analytic approach”, Social Science and Medicine, 64, págs. 712-724.

SCHWEDT, T. (2014): "Chronic Migraine”, BMJ, 348:g1416.

Surmann, V. (2005): Anfallsbilder. Metaphorische Konzepte im Sprechen anfallskranker Menschen, Würzburg, Königshausen \& Neumann. 


\section{APÉNDICE}

\section{CONVENCIONES DE TRANSCRIPCIÓN}

\begin{tabular}{|l|l|}
\hline$\cdot$ & Curva de entonación ascendente \\
\hline, & Curva de entonación descendente \\
\hline MAYÚSCULAS & Énfasis \\
\hline$:$ & Alargamiento de un sonido / una sílaba \\
\hline$/$ & Interrupción perceptible, corrección \\
\hline$=$ & Unión perceptible \\
\hline$*$ & Emisiones superpuestas \\
\hline$\&$ & Encabalgamiento rápido de intervenciones \\
\hline$\ldots . .$. & Pausa: muy corta, corta, más extensa \\
\hline$(?$ palabra $)$ & Transcripción insegura \\
\hline$<$ Comentario $>+$ & El comentario es válido para el segmento hasta el signo + \\
\hline
\end{tabular}

